\title{
Analysis and compensation of active power measurement error caused by non-integer period sampling
}

\author{
Hui Peng ${ }^{\mathrm{a}}$,Yan-Jie Song ${ }^{\mathrm{a}^{*}}$, Shao-Teng $\mathrm{Li}^{\mathrm{b}}$ \\ ${ }^{a}$ Wuhan University, Wuhan 430072, China \\ ${ }^{b}$ State Grid Zhejiang Electric Power Company, Electric Power Research institute, Hangzhou 310014, China
}

\begin{abstract}
A software compensation method is proposed in this paper to reduce the active power measurement error which is caused by non-integer period sampling in the electrical energy measurement process. In the process of electrical energy measurement, the sampling frequency is fixed, and it is an integer multiple of the standard frequency. When the grid frequency fluctuates, the real-time frequency deviates from the standard frequency, therefore, the sampling rate is not strictly the integer multiple of the grid frequency, which may lead to active power error. In this paper, we firstly calculate the analytic equation of active power error in non-integer period sampling, which includes the theory value and the error value, then the active power value of integral period sampling can be obtained by subtracting the error part. Finally, the MATLAB simulation shows the correction and effective of the compensation method, and it can significantly improve the accuracy of energy measurement.
\end{abstract}

Keywords: Active power, non-integer period sampling, error compensation, real-time frequency

\section{Introduction}

In power system, due to the fluctuation of the system frequency, the sampling frequency and signal frequency are out of synchronization, namely non-integer period sampling, and this may lead to active power error [1]. There are three traditional ways to solve this problem: 1) FFT interpolation algorithm; 2) synchronous device, such as digital phase locked loop and so on; 3) software algorithm based on sampling frequency self-adaptive techniques [2]. Among them, FFT interpolation algorithm can effectively reduce the error, but its computational complexity is too high; synchronous device can achieve frequency synchronization, but it may increase hardware complexity; software algorithm based method can get relatively accurately signal real-time frequency. By using this method, the sampling frequency can be adjusted dynamically to follow the real-time frequency, and the synchronous sampling can be realized, therefore, the synchronization error will be reduced.

As we all know, the sampling frequency of the electronic transformer is constant in the system of electrical energy measurement. Furthermore, according to the IEC 61850 protocol, the sampling value should be formed in the merging unit in which frequency value is not included; this means the sampling frequency could not be changed to achieve synchronous sampling based on the measured signal frequency. Therefore, the three traditional methods mentioned above cannot be applied to digital electrical energy meter.

To compensate the active power error caused by integer-period sampling, $\mathrm{Wu}$ [4] derived out a relevant modify formula and pointed out that the measurement error can be reduced by choosing appropriate sampling frequency, the sampling rate and the initial sampling time. Although Wu's method can reduce the measurement relative error, the relative error rate of the proposed method is still more than $0.2 \%$, which does not meet the requirements of $0.2 \mathrm{~s}$ electric energy meter. Xie [5] and $\mathrm{Yu}[6]$ derived out integer-period sampling error analysis equation in time domain and frequency domain respectively.

\footnotetext{
* Manuscript received May 9, 2014; revised July 21, 2014.

Corresponding author: Yan-Jie Song; Tel.: +86 15827359848; E-mail address: songpandejie@ 163.com.

doi: 10.12720/sgce.4.1.65-71
} 
Vasconcellos[7], Phadke[8] and Wang [9] analyzed the frequency and phase angle measurement error caused by non-integer period sampling. But all these methods can not solve the compensation problem.

In this paper, a software compensation method is proposed to reduce the active power measurement error. The proposed method firstly calculates the analytic equation of active power error in non-integer period sampling, then, by subtracting the error part, the active power value of integral period sampling can be obtained. MATLAB simulation results show that our proposed method can measure the electric energy more accurately than other methods.

\section{Analysis and Compensation of Active Power Measurement Error}

\subsection{Numerical sampling analysis}

The grid voltage and current signal are given by (1) and (2):

$$
\begin{aligned}
& u(t)=U \cos \left(2 \pi f t+\varphi_{u}\right) \\
& i(t)=I \cos \left(2 \pi f t+\varphi_{i}\right)
\end{aligned}
$$

where $f$ is the frequency of standard voltage and current, and $T=1 / f$ is the signal period.

Sampling frequency is constant in the digital electric energy meter system. Set sampling point $N$ in a period, signal sampling frequency is $f_{s}=N f$, signal sampling period is $T_{s}=1 / f_{s}$. The sampling sequence of the voltage, current signal can be obtained as follows:

$$
\begin{aligned}
& u(n)=U \cos \left(2 \pi f T_{S} n+\varphi_{u}\right) \\
& i(n)=I \cos \left(2 \pi f T_{S} n+\varphi_{i}\right)
\end{aligned}
$$

According to the formula of active power, we can get the active power by following equation:

$$
P=\frac{1}{T} \int_{0}^{T} u(t) i(t) d t=\frac{1}{N} \sum_{n=1}^{N} u(n) i(n)
$$

According to the sampling theorem, in order to make the band limited signal be sampled to completely recover, the sampling frequency must be more than twice the maximum frequency of the analog signal being measured [10]. The measurement result is accurate if it meets the sampling theorem and it is integer-period sampling.

\subsection{Non-integer period sampling error analysis}

In the operation of power system, if the total power demand and supply keep balance, the grid frequency will be $50 \mathrm{~Hz}$, and the frequency value will not change over time. However, the electric load and the electricity generated by generator changed frequently in reality. Once the balance is broken, the frequency will fluctuate and deviate from the standard frequency.

The purpose of the integer-period sampling is to ensure that the truncation interval after sampled is exactly integer multiple of the signal period. The active power can be obtained by following equation:

$$
P=\frac{1}{T} \int_{0}^{T} u(t) i(t) d t
$$

where $T$ is the signal period. When the frequency is not $50 \mathrm{~Hz}$, the active power value calculated by (6) is not periodic, and the measurement error will emerge.

\subsection{The calculation of analytic formulas}

In order to calculate the measurement error correctly and provide a basis for the compensation of measurement error, we deduce out the calculation formulas of the active power. And the process is as 
follows:

Set $f^{\prime}$ is the real-time frequency, $f$ is the standard frequency, $\Delta f$ is the difference, we can get $f^{\prime}=f+\Delta f$. Set relative difference is $\alpha=\Delta f / f$, based on equation (3), (4), and (5), we can get a period active power by following equation:

$$
\begin{aligned}
P=\frac{1}{N} \sum_{n=0}^{N-1} u(n) i(n) & =\frac{1}{N} \sum_{n=0}^{N-1} U \cos \left(2 \pi f T_{S} n+\varphi_{u}\right) I \cos \left(2 \pi f T_{S} n+\varphi_{i}\right) \\
& =\frac{1}{N} \sum_{n=0}^{N-1} U \cos \left(2 \pi \frac{1+\alpha}{N} n+\varphi_{u}\right) I \cos \left(2 \pi \frac{1+\alpha}{N} n+\varphi_{i}\right)
\end{aligned}
$$

If $\alpha=0$, it means that the sampling is based on a full period, we can get the following equations:

$$
P=\frac{1}{N} \sum_{n=0}^{N-1} U \cos \left(2 \pi \frac{n}{N}+\varphi_{u}\right) I \cos \left(2 \pi \frac{n}{N}+\varphi_{i}\right)=\frac{1}{2} U I \cos \left(\varphi_{u}-\varphi_{i}\right)=P_{0}
$$

If $\alpha \neq 0$, it means that the sampling is not based on a full period, we can get the following equations:

$$
\begin{aligned}
P & =\frac{1}{N} \sum_{n=0}^{N-1} u(n) i(n)=\frac{1}{N} \sum_{n=0}^{N-1} U \cos \left(2 \pi f T_{S} n+\varphi_{u}\right) I \cos \left(2 \pi f T_{S} n+\varphi_{i}\right) \\
& =\frac{1}{2} U I \cos \left(\varphi_{u}-\varphi_{i}\right)+\frac{U I}{2 N} \cos \left(2 \pi\left(\alpha-\frac{1+\alpha}{N}\right)+\varphi_{u}+\varphi_{i}\right) \cdot \frac{\sin (2 \alpha \pi)}{\sin \left(2 \pi \frac{1+\alpha}{N}\right)}=P_{0}+P_{0}^{\prime}
\end{aligned}
$$

where

$$
\begin{aligned}
& P_{0}=\frac{1}{2} U I \cos \left(\varphi_{u}-\varphi_{i}\right) \\
& P_{0}^{\prime}=\frac{U I}{2 N} \cos \left(2 \pi\left(\alpha-\frac{1+\alpha}{N}\right)+\varphi_{u}+\varphi_{i}\right) \cdot \frac{\sin (2 \alpha \pi)}{\sin \left(2 \pi \frac{1+\alpha}{N}\right)}
\end{aligned}
$$

where $P_{0}$ is the active power of a period, $P_{0}^{\prime}$ is the measurement error caused by non-integer period sampling.

According to equations (9), (10) and (11), we can get the relative error of active power in the process of non-integer period sampling:

$$
e=\left|\frac{P-P_{0}}{P_{0}}\right| \times 100 \%=\left|\frac{P_{0}^{\prime}}{P_{0}}\right| \times 100 \%
$$

From equations (11) we can see that the measurement error is related to real-time frequency. Once the real-time frequency is provided, we can compute the measurement error and make compensation for it.

\subsection{Real-time frequency measurement method}

Unlike the traditional energy meter, there is no frequency information in the digital energy meter system, and we can only calculate the frequency based on the voltage and current value of a period. There are two frequency measurement methods widely used, namely "analytic method" and "zero-crossing detection method" [11]. The former calculates the frequency by capturing any two points for the same interval in a period, it is very complex. The latter calculates the frequency by detecting the adjacent position of zero value, it is simple and accuracy. In this paper, we propose to use "Zero-crossing Detection Method" to calculate the real-time frequency. 
Fig. 1 shows the diagram of zero-crossing detection method.

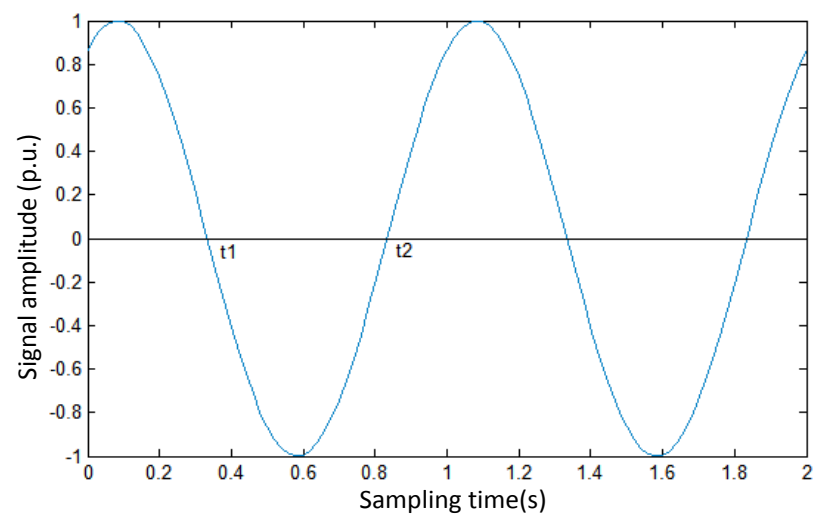

Fig. 1. Diagram of zero-crossing detection method

From Fig. 1, we can see the signal frequency can be obtained by the time of zero-crossing $t_{1}$ and $t_{2}$ :

$$
f=\frac{1}{T}=\frac{1}{2\left(t_{2}-t_{1}\right)}
$$

For a discrete-time signal, the zero-crossing point may be not included in the series of sampling point. According to the theory that a sinusoidal signal can be linearized around zero, we can obtain the zerocrossing point. Supposed that the power series of sine function is $\sin x=x-\frac{x^{3}}{3 !}+\frac{x^{5}}{5 !}-\frac{x^{7}}{7 !}+\cdots$, when $x$ is a very small value near zero, the high-order term can be ignored, the function can be approximate as $\sin x \approx x$. In a discrete-time system, the more sampling points, the better linearization of the function around zero. Supposed that the two points are $\left(x_{1}, x_{2}\right)$ and $\left(y_{1}, y_{2}\right)$ respectively, the zero-crossing point can be calculated based on the two-point near zero (see Fig. 2).

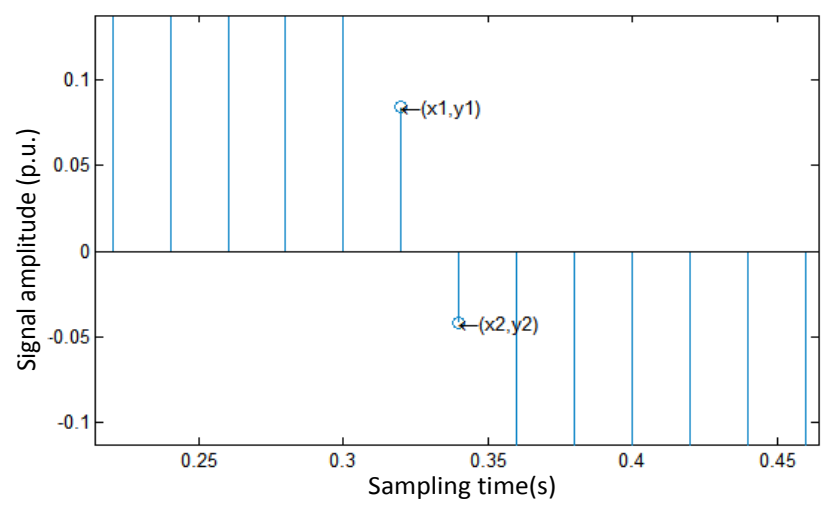

Fig. 2. Diagram of zero-crossing point calculation.

The linear equation around zero can be obtained by follows:

$$
y=\frac{y_{2}-y_{1}}{x_{2}-x_{1}}\left(x-x_{1}\right)+y_{1}
$$

Set $y=0$, we can obtain the time of zero-crossing point by follows:

$$
t_{1}=x_{1}-y_{1} \frac{x_{2}-x_{1}}{y_{2}-y_{1}}
$$


And $t_{2}$ can be obtained in the same way.

Given two points around zero, the zero-crossing position can be calculated theoretically by using zerocrossing detection method. However, calculation error will emerge if there are noises and disturbances existed in the electric system. In order to deal with the noise and disturbances problem effectively, we propose to statistic the multipoint around the zero point [12]. The merits of zero-crossing detection method are as follows:

1) In the digital electrical energy meter system, the number of sampling point in a period is 256 . The more the sampling points, the higher the accurate of the linearization results and the frequency;

2) The method can calculate the frequency according to the value of voltage and current in a period transferred from the merging unit, and it needs no additional hardware;

3) By using the traditional way, we should change the frame format based on IEC 61850 to calculate the frequency, however, the zero-crossing detection method need not to change the data frame format.

\subsection{The process of non-integer period sampling error compensation}

The compensation steps for non-integer period sampling error are as follows:

Step 1: Calculate the active power $P$ and the real-time frequency $f^{\prime}$ based on the value of voltage and current in a period transferred from the merging unit;

Step 2: Calculate the active power error $\Delta P$ introduced in non-integer period sampling according to equations (11);

Step 3: After compensation, we can get $P^{\prime}=P-\Delta P$.

\section{Simulation Based on Matlab}

\subsection{Simulation of numerical sampling}

We simulate the active power error under different sampling rate in integer period sampling system. Set the voltage $u=57.7 \sqrt{2} \cos (2 \pi f t+\pi / 3) \mathrm{V}$, the current $i=3 \sqrt{2} \cos (2 \pi f t) \mathrm{A}$, and the sampling frequency $f_{s}=256 \times 50 \mathrm{~Hz}$. The sampling point in a period is 256 . If the real-time frequency is $50 \mathrm{~Hz}$, the result of active power simulation is shown in Table 1 .

Table 1. Integer period sampling relative error in different sampling rate

\begin{tabular}{cccc}
\hline \hline Sampling rate $(\mathrm{Hz})$ & Theoretical value of active power $(\mathrm{W})$ & Calculated value of active power $(\mathrm{W})$ & Relative error \\
\hline 2500 & 86.55 & 86.55 & $2.22 \mathrm{E}-16$ \\
3200 & 86.55 & 86.55 & $4.44 \mathrm{E}-16$ \\
5000 & 86.55 & 86.55 & $6.66 \mathrm{E}-16$ \\
6400 & 86.55 & 86.55 & $4.44 \mathrm{E}-16$ \\
10000 & 86.55 & 86.55 & $6.66 \mathrm{E}-16$ \\
12800 & 86.55 & 86.55 & $-2.22 \mathrm{E}-16$ \\
\hline \hline
\end{tabular}

From Table 1 we can see that when the sampling rate is the integer multiple of the standard frequency, the measurement error is very small, and it can be ignored.

\subsection{Simulation of non-integer period sampling}

In order to obtain the error value in non-integer period sampling system and test the effect of the proposed compensation method, we simulate the proposed method on Matlab. The results are shown in Fig. 3 to Fig. 5.

Fig. 3 shows that result of active power relative error in non-integer period sampling system. From Fig. 3 we can see the relative error reach up to $0.4 \%$ at $49.8 \mathrm{~Hz}$, which is far beyond the requirement of $0.2 \mathrm{~s}$ digital electric energy meter. The error in non-integer period sampling system is so big that we can not ignore it. 
Fig. 4 shows the results of frequency calculation relative error curve. From Fig. 4 we can see the relative error is smaller than $4 \times 10^{-6}$. It means the zero-crossing detection method is a good way to calculate the real-time frequency.

Fig. 5 shows the result of active power relative error after compensation. From Fig. 5 we can see the relative error is drastically reduced, and it can meet the requirement of $0.2 \mathrm{~s}$ digital electric energy meter. Therefore, the method presented in this paper can effectively improve the accuracy of the electric energy metering.

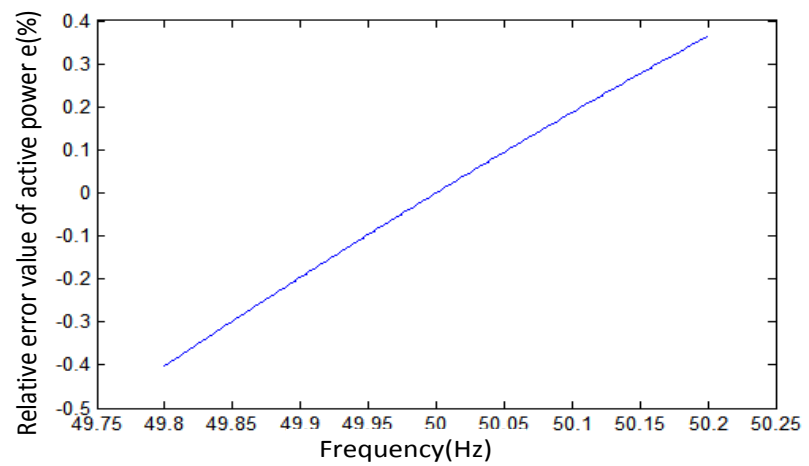

Fig. 3. Active power relative error in non-integer period sampling system.

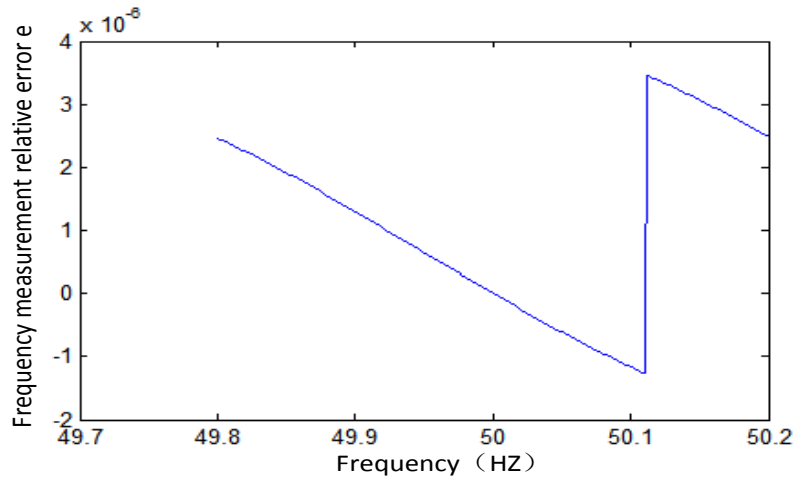

Fig. 4. Frequency calculation relative error curve.

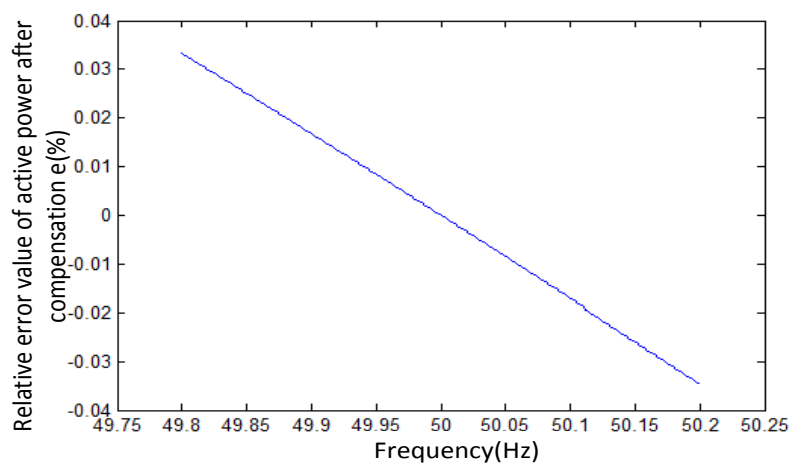

Fig. 5. Active power relative error curve after compensation.

\section{Conclusion}

In the digital electrical energy metering system, the electronic transformer adopts fixed value sampling, and the sampling rate is the integer multiple of the standard frequency. When the grid frequency fluctuates overtime, the active power error will emerge. 
In order to reduce the active power measurement error, this paper proposed an error compensation algorithm based on zero-crossing detection method. The proposed method firstly calculates the analytic equation of active power error in non-integer period sampling, which includes the theory value and the error value; then, by subtracting the error part the active power value of integral period sampling can be obtained. Experimental results show that the method can significantly improve the accuracy of energy measurement. Furthermore, the proposed method need not add hardware device and change the IEC 61850 protocol format. Although it is simple, it provides effective theoretical references for engineering application.

\section{References}

[1] Zhang B, Kong M, Wu CB. Research of spectrum leakage with window function. Informatization, 2009, (11):10-12.

[2] Yang FK. The research of harmonic detection methods in grid based in FFT. Degree dissertation. Xian University of Science and Technology. 2009.

[3] Communication Networks and Systems in Substations. IEC 61850-9, FDIS, 2003.

[4] Wu J, Zhao W. Error analysis for electrical power measurements with non-integer-period sampling. J Tsinghua Univ.(Sci\&Tech), 2006; 46(7):1197-1204.

[5] Xie D, Xie P, Fu Z. Error analysis of non-integer-period sampling for electric sinusoidal signal. Journal of Electronic Measurement and Instrument, 2010; 24(2):113-118.

[6] Yu J, Liu J, Zheng K. Analysis for harmonic active power measurement with non-integer-period sampling in time-frequency domain. Computer simulation, 2011; 28(1):307-339.

[7] Barros e Vasconcellos, RT, Compos MLR. Sampling error analysis applied to high-accuracy measurement. Presented at: CPEM. 2008

[8] Phadke AG, Thorp JS, Adamiak MG. A new measurement technique for tracking voltage phases, local system frequency, and rate of change of frequency. IEEE Transactions on Power Apparatus and System, May 1983; 5:10025-1038.

[9] Wang M, Sun Y. A practical, precise method for frequency tracking and phase estimation. IEEE Trans on Power Delivery, 2004; 19(4):1547-1552.

[10] Hu P, Zhang Y, Wang S. Signals and Systems. Beijing: Chinese Electric Power Press; 2009.

[11] Li J. Development of digital multifunction electrical energy metering system. Degree dissertation. North China Electrical Power University. 2008.

[12] Yi LQ. A digital sinusoidal signal frequency measurement algorithm based on linear regression analysis theory. Electrical Measurement \& Instrumentation, 2008; 48(3):20-24. 